\title{
Discovery of Water Maser Emission in Five AGNs and a Possible Correlation Between Water Maser and Nuclear 2-10 keV Luminosities
}

\section{Citation}

Kondratko, Paul T., Lincoln J. Greenhill, and James M. Moran. 2006. “Discovery of Water Maser Emission in Five AGNs and a Possible Correlation Between Water Maser and Nuclear 2-10 keV Luminosities." The Astrophysical Journal 652 (1) (November 20): 136-145. doi:10.1086/507885.

\section{Published Version}

$10.1086 / 507885$

\section{Permanent link}

http://nrs.harvard.edu/urn-3:HUL.InstRepos:32095372

\section{Terms of Use}

This article was downloaded from Harvard University's DASH repository, and is made available under the terms and conditions applicable to Other Posted Material, as set forth at http:// nrs.harvard.edu/urn-3:HUL.InstRepos:dash.current.terms-of-use\#LAA

\section{Share Your Story}

The Harvard community has made this article openly available.

Please share how this access benefits you. Submit a story.

\section{Accessibility}




\title{
Discovery of Water Maser Emission in Five AGN and a Possible Correlation Between Water Maser and Nuclear 2-10 keV Luminosities
}

\author{
Paul T. Kondratko, Lincoln J. Greenhill, James M. Moran \\ Harvard-Smithsonian Center for Astrophysics, 60 Garden St., Cambridge, MA 02138, USA \\ pkondrat@cfa.harvard.edu
}

\begin{abstract}
We report the discovery of water maser emission in five active galactic nuclei (AGN) with the 100-m Green Bank Telescope (GBT). The positions of the newly discovered masers, measured with the VLA, are consistent with the optical positions of the host nuclei to within $1 \sigma\left(0^{\prime \prime} 3\right.$ radio and 1"'3 optical) and most likely mark the locations of the embedded central engines. The spectra of three sources, 2MASX J08362280+3327383, NGC 6264, and UGC 09618 NED02, display the characteristic spectral signature of emission from an edge-on accretion disk with maximum orbital velocity of $\sim 700 \mathrm{~km} \mathrm{~s}^{-1}, \sim 800 \mathrm{~km} \mathrm{~s}^{-1}$, and $\sim 1300 \mathrm{~km} \mathrm{~s}^{-1}$, respectively. We also present a GBT spectrum of a previously known source MRK 0034 and interpret the narrow Doppler components reported here as indirect evidence that the emission originates in an edge-on accretion disk with orbital velocity of $\sim 500 \mathrm{~km} \mathrm{~s}^{-1}$. We obtained a detection rate of $12 \%$ (5 out of 41 ) among Seyfert 2 and LINER systems with $10000 \mathrm{~km} \mathrm{~s}^{-1}<v_{\text {sys }}<15000 \mathrm{~km} \mathrm{~s}^{-1}$. For the 30 nuclear water masers with available hard X-ray data, we report a possible relationship between unabsorbed X-ray luminosity $(2-10 \mathrm{keV})$ and total isotropic water maser luminosity, $L_{2-10} \propto L_{\mathrm{H}_{2} \mathrm{O}}^{0.5 \pm 0.1}$, consistent with the model proposed by Neufeld and Maloney in which X-ray irradiation and heating of molecular accretion disk gas by the central engine excites the maser emission.
\end{abstract}

Subject headings: galaxies: active - galaxies: individual (2MASXJ08362280+3327383, NGC6264, UGC09618NED02, IRAS03355+0104, SBS0927+493, MRK0034, NGC3393, NGC5495, VIIZW073, IC0184, AM2158-380NED02) — galaxies: Seyfert — ISM: molecules - ISM: jets and outflows - masers 


\section{Introduction}

Water maser emission $(\lambda=1.3 \mathrm{~cm})$ is currently the only resolvable tracer of warm dense molecular gas in the inner parsec of active galactic nuclei (AGN) and has been detected to date in approximately 60 nuclei, the great majority of which are classified optically as Seyfert 2 or LINER (e.g., Braatz et al. 1996, 2004; Greenhill et al. 2003; Henkel et al. 2005; Kondratko et al. 2006; Zhang et al. 2006). Because of the association of maser emission with nuclear activity, X-ray irradiation of molecular gas by the central engine provides the most likely model for exciting the maser emission (e.g., Neufeld, Maloney, \& Conger 1994). Maser emission might be associated with Seyfert 2 systems in particular because, over a range of AGN luminosity, the shielding column density that provides the obscuring geometry in type 2 AGN (e.g., Lawrence \& Elvis 1982; Antonucci 1993) maintains not only a reservoir of molecular gas but also physical conditions conducive to maser action, which are temperatures of $250-1000 \mathrm{~K}$ and $\mathrm{H}_{2}$ number densities of $10^{8-10} \mathrm{~cm}^{-3}$ (e.g., Desch, Wallin, \& Watson 1998). The importance of the obscuring geometry in this context is supported by an empirical observation that water maser sources are found preferentially in nuclei with large hydrogen column densities $\left(N_{H}>10^{24} \mathrm{~cm}^{-2}\right.$; Braatz et al. 1997b; Madejski et al. 2006; Zhang et al. 2006). There is good evidence that LINERs are low-luminosity analogues of Seyfert 2 systems (e.g., Ho et al. 1997; Ho 1999a; Ho et al. 2003), which might explain the association of maser emission with the former. If X-ray irradiation indeed excites the maser emission (e.g., Neufeld et al. 1994), then a relationship between X-ray and water maser luminosities might reflect this

dependance. Braatz et al. (1997b) observed no correlation between the two, but that study was based on only seven water maser systems and relied on the relatively coarse X-ray data from EXOSAT, GINGA, and ASCA telescopes, with which luminosities can be difficult to estimate accurately when column densities are large. On the other hand, Henkel et al. (2005) reported a correlation between infrared and total isotropic water maser luminosities (i.e., assuming the isotropic emission of maser radiation), and this might be indirectly indicative of a physical relationship between X-ray and maser luminosities, mediated by dust reprocessing (e.g., Franceschini et al. 2005). The substantial increase in the number of known water maser sources over the past few years (e.g., Greenhill et al. 2003; Braatz et al. 2004; Kondratko et al. 2006) combined with the recent growth in the number of AGN for which high-quality $\mathrm{X}$-ray spectra have been obtained (with angular apertures that isolate the central engines reasonably well), have enabled a new look at the possibility of a relationship between X-ray and water maser luminosities.

Very Long Baseline Interferometry (VLBI) maps of seven water maser sources have been interpreted in a context of a model in which the maser emission traces a nearly edge-on disk of molecular material 0.1 to 1 pc from a supermassive black hole: NGC 4258 (Miyoshi et al. 1995), NGC 1386 (Braatz et al. 1997a), NGC 4945 (Greenhill et al. 1997), NGC 1068 
(Greenhill \& Gwinn 1997), NGC 3079 (Trotter et al. 1998), IC 2560 (Ishihara et al. 2001), and Circinus (Greenhill et al. 2003). As a consequence of these studies, it is believed that maser emission is detected preferentially in edge-on parsec-scale disks along the diameter perpendicular to the line of sight (a.k.a. the midline) and close to the line-of-sight towards the center. These are the loci within disks where the gradient in line-of-sight velocity is zero and, as a result, the coherent paths for maser emission are maximized. Thereby, a characteristic spectral signature of emission from an edge-on disk consists of a spectral complex in the vicinity of the systemic velocity (low-velocity emission) and two spectral complexes (highvelocity emission) more or less symmetrically offset from the systemic velocity by the orbital velocity ( $>100 \mathrm{~km} \mathrm{~s}^{-1}$, based on aforementioned VLBI studies). Sources that display such spectra constitute approximately $40 \%$ of the known nuclear water masers and are referred to here as high-velocity systems.

Discovery of new high-velocity water maser systems is important because VLBI maps of these sources can be used to determine pc-scale accretion disk structures, to estimate accurately black hole masses (e.g., Greenhill \& Gwinn 1997; Greenhill et al. 2003; Herrnstein et al. 2005), and to obtain distances to the host galaxies independent of standard candles (Herrnstein et al. 1999), the latter in cases where a robust disk model is combined with a measurement of either maser proper motions or drifts in the line-of-sight velocities of spectral features (i.e., centripetal acceleration). However, surveys for water maser emission are challenging and require high sensitivity, wide bandwidth, and high spectral resolution because 1 ) the water maser emission is weak ( $\ll 1 \mathrm{Jy}), 2$ ) its velocity extent is determined by the rotational velocity of the accretion disk, which can exceed $1000 \mathrm{~km} \mathrm{~s}^{-1}$ and is not known in advance, and 3) the maser lines are typically narrow $\left(\leq 1 \mathrm{~km} \mathrm{~s}^{-1}\right)$. The Green Bank Telescope (GBT) of the $\mathrm{NRAO}^{1}$ and its wide-bandwidth spectrometer together constitute the most sensitive observing system currently in operation at $\lambda=1.3 \mathrm{~cm}$; as a result, detection rates for samples with comparable distributions of distance are highest for surveys conducted with the GBT. For instance, the seminal survey of AGN with $1 \sigma \sim 60 \mathrm{mJy}$ sensitivity (in $\sim 1 \mathrm{~km} \mathrm{~s}^{-1}$ spectral channels) by Braatz et al. (1996) and a search with $1 \sigma \sim 14 \mathrm{mJy}$ sensitivity (in $1.3 \mathrm{~km} \mathrm{~s}^{-1}$ spectral channels) with the 70-m NASA Deep Space Network (DSN) antennas (Greenhill et al. 2003; Kondratko et al. 2006) yielded incidence rates of water maser emission among nearby $\left(v_{\text {sys }}<7000 \mathrm{~km} \mathrm{~s}^{-1}\right)$ Seyfert 2 and LINER systems of $\sim 7 \%$ and $\sim 10 \%$, respectively. However, a GBT survey conducted with $1 \sim 3$ mJy sensitivity (converted to $1.3 \mathrm{~km} \mathrm{~s}^{-1}$ spectral channels) resulted in an incidence rate of $\sim 20 \%$ among Seyfert 2 and LINER systems with $v_{\text {sys }}<7500 \mathrm{~km} \mathrm{~s}^{-1}$ (Braatz et al. 2004).

\footnotetext{
${ }^{1}$ The National Radio Astronomy Observatory is operated by Associated Universities, Inc., under cooperative agreement with the National Science Foundation
} 
We used the GBT to survey 56 AGN with $10000<v_{\text {sys }}<30000 \mathrm{~km} \mathrm{~s}^{-1}$ selected from the NASA Extragalactic Database (NED). Previous surveys have concentrated on detection of water maser emission mostly among nearby sources (cf. Henkel et al. 1998; Barvainis \& Antonucci 2005). In particular, galaxies with $v_{\text {sys }}>10000 \mathrm{~km} \mathrm{~s}^{-1}$ constitute only $\sim 7 \%$ and $14 \%$ of AGN in the two largest surveys to date (Braatz et al. 1996 and Kondratko et al. 2006, respectively) and, as a result, only 4 out of $\sim 60$ known maser sources lie beyond $10000 \mathrm{~km} \mathrm{~s}^{-1}$ (Tarchi et al. 2003; Henkel et al. 2005; Kondratko et al. 2006, Zhang et al. 2006). We have discovered water maser emission in five AGN between 10000 and $15000 \mathrm{~km} \mathrm{~s}^{-1}$. In this paper, Section 3, we present spectra and sub-arcsecond positions for the detected emission and address survey statistics. In Section 4, we discuss a possible correlation between X-ray and water maser luminosities.

\section{Observations}

The survey was conducted during the 2003-2004 northern winter with the GBT using the observing system and correlator setup identical to that described in Braatz et al. (2004). The channel spacing was $24.4 \mathrm{kHz}$ and the instantaneous bandwidth was $380 \mathrm{MHz}\left(\approx 5600 \mathrm{~km} \mathrm{~s}^{-1}\right.$ for a representative recessional velocity of $12500 \mathrm{~km} \mathrm{~s}^{-1}$, assuming the optical definition of Doppler shift). To obtain total-power spectra of each source, we nodded the telescope by $3^{\prime}$ every 2 min between two positions on the sky so that each target was always present in one of the two GBT beams for each polarization. System temperatures were measured against a calibrated noise source injected at the receiver and ranged from 28 to $59 \mathrm{~K}$ depending on elevation and weather conditions. By comparing maser line amplitudes among beams and polarizations, we estimate that the calibration of the system temperature is accurate to within $30 \%$. This uncertainty dominates the error budget for the flux density scale in the survey. Antenna pointing corrections - obtained every $\sim 30$ minutes using sources from the VLA Calibrator Manual - were typically $<6^{\prime \prime}$, which corresponds to a $<8 \%$ loss in source flux density for a $36^{\prime \prime}$ beamwidth (FWHM) at $1.3 \mathrm{~cm}$. For the observations reported here, the wind speed at the GBT site was generally $<13 \mathrm{mph}$, which corresponds to a one-dimensional rms tracking error of $<5^{\prime \prime}$ and a signal loss of $<6 \%$ (with the exception of CGCG 482-008 and MRK 0948, which were observed with wind gusts up to $20 \mathrm{mph}$, corresponding to a one-dimensional rms tracking error of $<13^{\prime \prime}$ and a signal loss of $<29 \%$; Condon 2003). Data were reduced using scripts written in the Interactive Data Language. We subtracted a running boxcar average of width $6.25 \mathrm{MHz}$ to remove systematic baseline structure from the total-power spectra. To convert the spectra to flux density units, we used the gain curve obtained by the GBT staff based on measurements of opacity corrected antenna temperature for NGC 7027 at $\sim 1.4 \mathrm{~cm}$ (R. Maddalena 2003, private communication). The resulting $1 \sigma$ 
noise levels attained in an integration of $\sim 30$ minutes total and corrected for atmospheric opacity estimated from tipping scans (typically $\sim 0.06$ ) were $3-6 \mathrm{mJy}$ in a $24.4 \mathrm{kHz}$ channel. The spectra presented here have been iteratively Hanning smoothed to an effective resolution of $108 \mathrm{kHz}$.

\section{Results}

In a survey with the GBT of $56 \mathrm{AGN}$ with $10000 \mathrm{~km} \mathrm{~s}^{-1}<v_{\text {sys }}<30000 \mathrm{~km} \mathrm{~s}^{-1}$ selected from the NED (Table 1), we have detected five new water maser sources: UGC09618 NED02, 2MASX J08362280+3327383, NGC 6264, IRAS 03355+0104, SBS 0927+493 (Fig. 1 and Table 2). The five discoveries were subsequently confirmed with the Very Large Array (VLA) using narrow observing bandwidths $(6.25-12.5 \mathrm{MHz})$. In addition, the UGC 09618 NED02, 2MASX J08362280+3327383, NGC 6264, and SBS 0927+493 broadband (i.e., $350 \mathrm{MHz}$ ) maser spectra were confirmed by at least one observation on a different day with the GBT. The nuclei that are host to the detected maser emission are spectroscopically classified as Seyfert 2 or LINER (Table 2). Positions of the maser emission measured with the VLA are consistent with optical positions of the AGN to within $1 \sigma$ (typically 0 "' 3 radio and $1^{\prime \prime} .3$ optical, or of order $1 \mathrm{kpc}$ at a recessional velocity of $15000 \mathrm{~km} \mathrm{~s}^{-1}$ ), which is suggestive of an association of the detected emission with nuclear activity.

The maser spectra of UGC 09618 NED02, 2MASX J08362280+3327383, and NGC 6264 show a characteristic spectral signature of emission from an edge-on disk: two high-velocity complexes approximately symmetrically offset from the systemic velocity and a third spectral complex in the vicinity of the systemic velocity. If the highly red- and blue-shifted emission in these systems indeed represents high-velocity emission, then the maximum orbital velocities of the disks as traced by the maser emission are $\sim 1300 \mathrm{~km} \mathrm{~s}^{-1}, \sim 700 \mathrm{~km} \mathrm{~s}^{-1}$, and $\sim 800 \mathrm{~km} \mathrm{~s}^{-1}$, respectively, making UGC 09618 NED02 the fastest known rotator. (The previously fastest rotator was NGC 4258 at $\sim 1200 \mathrm{~km} \mathrm{~s}^{-1}$; Modjaz et al. 2005.) Because the high-velocity emission extends over $\Delta v \sim 750 \mathrm{~km} \mathrm{~s}^{-1}$ for UGC 09618 NED02, it must occupy a fractional range of radii $\Delta r / R \approx 2 \Delta v / v \sim 1.6$ assuming Keplerian rotation (where we have differentiated the Keplerian rotation law, $v \propto r^{-0.5}$, with respect to $r$ ). For a range in disk sizes from that of NGC $4258(0.16-0.28$ pc) to NGC $1068(0.6-1.1 \mathrm{pc})$, the maser emission in this source traces a disk of radial extent $\Delta r \sim 0.36-1.4 \mathrm{pc}$. The corresponding central mass would be $(6-24) \times 10^{7} M_{\odot}$, which is larger than black hole masses measured with VLBI to date (the highest is NGC 4258 at $3.9 \times 10^{7} M_{\odot}$ Herrnstein et al. 2005). The anticipated centripetal acceleration - that is the secular velocity drift of the systemic feature - would be $0.3-11 \mathrm{~km} \mathrm{~s}^{-1} \mathrm{yr}^{-1}$, which should be readily detectable within one year using single dish 
monitoring. The remaining two detections — IRAS 03355+0104 and SBS 0927+493 — display spectral feature(s) only in the vicinity of the systemic velocity of the host galaxy, which makes physical interpretation difficult.

We also used the GBT to obtain high signal-to-noise-ratio (SNR) spectra of known sources, including those recently discovered with the DSN (cf. Henkel et al. 2005; Kondratko et al. 2006; Fig. 2 and Table 3). Spectra of MRK 0034, NGC 3393, NGC 5495, VII ZW 073, and IC 0184 presented here are the most sensitive obtained to date (cf. Henkel et al. 2005; Kondratko et al. 2006). The narrow Doppler components in the spectrum of MRK 0034 reported here were not evident in the previous spectra of the source obtained with $4.65 \mathrm{~km} \mathrm{~s}^{-1}$ channels. Absent narrow lines, Henkel et al. (2005) suggested the possibility that the maser emission in MRK0034 might be excited by jet activity, which has been associated with broad spectral features in other AGN (e.g., Claussen et al. 1998; Gallimore et al. 2001; Peck et al. 2003). However, the new spectrum adds support for the hypothesis that MRK 0034 is a high-velocity system. In this context and considering the large uncertainty on the recessional velocity of the galaxy (see Fig. 2), the two spectral complexes are consistent with blue- and red-shifted high-velocity emission that is symmetrically displaced from the systemic velocity by $\sim 500 \mathrm{kms}^{-1}$. The GBT spectrum of NGC 3393 shows a new systemic complex at $\sim 3750 \mathrm{~km} \mathrm{~s}^{-1}$ as well as weak $(\sim 5 \mathrm{mJy})$ high-velocity lines at $\sim 3098, \sim 3293$, $\sim 3396, \sim 4190$, and $\sim 4475 \mathrm{~km} \mathrm{~s}^{-1}$ (Fig. 2). We also confirmed the low-velocity line at $\sim 3878 \mathrm{~km} \mathrm{~s}^{-1}$ in the NGC 3393 spectrum and the high-velocity lines in the NGC 5495 spectrum, which were all marginally detected with the DSN (Kondratko et al. 2006).

Our detection rate is consistent with the survey being sensitivity limited. Among Seyfert 2 and LINER systems with $10000 \mathrm{~km} \mathrm{~s}^{-1}<v_{\text {sys }}<15000 \mathrm{~km} \mathrm{~s}^{-1}$, we obtain a detection rate of 5 out of 41 or $12 \% \pm 5 \%$, which should be compared to the incidence rates of $20 \% \pm 5 \%$ (15 out of 75 ) and $14 \% \pm 5 \%$ ( 7 out of 49 ) in velocity bins $0-5000$ and $5000-12000 \mathrm{~km} \mathrm{~s}^{-1}$, respectively, from a GBT survey of the same sensitivity (Braatz et al. 2004; to estimate the errors, we used the unbiased maximum likelihood estimator for the variance of the Binomial parameter $p$ ). The detection rate decreases with distance because the spectral rms noise level was approximately the same for each source in both surveys. The detection rate of high-velocity emission is $60 \% \pm 24 \%$ ( 3 out of 5 ) in this survey or $44 \% \pm 13 \%$ (7 out of 16) if we include the GBT detections reported in Braatz et al. (2004; where we excluded the starburst galaxy NGC 2782). These rates should be compared to the analogous rate of $27 \% \pm 12 \%$ (4 out of 15 ) in the DSN survey (Greenhill et al. 2003; Kondratko et al. 2006). Although the uncertainties due to counting statistics are large, the higher detection rates for high-velocity emission in the GBT surveys may have been a consequence of higher sensitivity, and perhaps finer spectral resolution as well. 


\section{Discussion}

Using the sample of known nuclear masers with available $>2 \mathrm{keV}$ X-ray data, we have identified a possible relationship between unabsorbed X-ray luminosity $(2-10 \mathrm{keV})$ and integrated water maser luminosity, assuming isotropic emission of radiation. In log-log space (Fig. 3 and Table 4), we obtain a slope of $m=0.4 \pm 0.1$ and a Spearman correlation coefficient of $\rho=0.4 \pm 0.1$ (Table 5). The probability that a random data set of the same size would yield a larger magnitude of the correlation coefficient (hereafter referred to as the significance level) is $0.07 \pm 0.07$.

Under the assumption of a thin viscous accretion disk obliquely illuminated at an angle $\cos ^{-1} \mu$ by a central X-ray source, the outer radius at which the disk becomes atomic and the maser emission ceases is given by $R_{c r} \propto L_{2-10}^{-0.43}(\dot{m} / \alpha)^{0.81} \mu^{-0.38} M_{B H}^{0.62}$ (Neufeld \& Maloney $1995)$, where $L_{2-10}$ is the $2-10 \mathrm{keV}$ luminosity, $M_{B H}$ is the mass of the central black hole, $\dot{m}$ is the mass accretion rate, and $\alpha$ is the standard dimensionless viscosity parameter (Shakura \& Sunyaev 1973). If we assume that X-ray heated accretion disks emit water maser radiation with a surface luminosity of $\sim 10^{2 \pm 0.5} L_{\odot} \mathrm{pc}^{-2}$ (and hence $L_{\mathrm{H}_{2} \mathrm{O}} \propto R_{c r}^{2}$; Neufeld et al. 1994), that $2-10 \mathrm{keV}$ luminosity is a fraction $\gamma$ of AGN bolometric luminosity $\left(L_{2-10}=\gamma L_{B o l}\right)$, that central engines radiate with an Eddington ratio $\eta\left(L_{B o l}=\eta L_{E d d} \propto \eta M_{B H}\right)$, and that they convert rest mass to energy with an accretion efficiency $\epsilon=L_{B o l} / \dot{m} c^{2}(\epsilon \sim 0.1$; Frank, King, \& Raine 2002), then we obtain $L_{2-10} \propto L_{\mathrm{H}_{2} \mathrm{O}}^{0.5}\left[(\epsilon \alpha)^{0.81} \gamma^{1.4} \mu^{0.38} \eta^{0.62}\right]$, in agreement with the observed trend.

The scatter about the linear relationship in Fig. 3 in excess of the reported uncertainties could be attributed to differences in the parameters $\alpha, \epsilon, \gamma, \mu$, and $\eta$ from source to source. For instance, although $0.01 \lesssim \gamma \lesssim 0.03$ based on an average quasar spectral energy distribution (SED; Fabian \& Iwasawa 1999; Elvis et al. 2002), the actual variation in $\gamma$ among individual Seyfert systems is more than an order of magnitude (e.g., $0.01<\gamma<0.6$; Kuraszkiewicz et al. 2003). Furthermore, although $\eta$ is relatively constrained for Seyfert nuclei (e.g., $0.01 \lesssim \eta \lesssim 1$; Padovani 1989; Wandel et al. 1999; Satyapal et al. 2005), it might be as low as $10^{-6.7}$ for LINER systems (e.g., Satyapal et al. 2005). For the LINER in NGC 4258 where black hole mass is well constrained with VLBI, we obtain $10^{-3.6}<\eta<10^{-2.9}$, where we adopted $M_{B H}=3.9 \times 10^{7} M_{\odot}$ (Herrnstein et al. 1999), $L_{2-10}=10^{40.6-41.2} \mathrm{erg} \mathrm{s}^{-1}($ Table 4 ), and $\gamma=0.03$ as determined from SEDs of 7 low-luminosity AGNs (Ho 1999b). Because of the broader range of Eddington ratio specific to LINERs, we have considered a Seyfert-only sub-sample. Furthermore, we have also removed galaxies in which the maser sources are associated with jets (for which the Neufeld \& Maloney model does not apply), and galaxies with multiple nuclei (wherein it may be uncertain which nucleus is responsible for the maser emission or the production of maser luminosity may in some way be affected by processes 
specific to mergers). The active galaxies with multiple nuclei are MRK 266 (e.g., Wang et al. 1997), ARP 299 (e.g., Ballo et al. 2004), NGC 6240 (e.g., Lira et al. 2002), and MRK 1066 (e.g., Gimeno et al. 2004). If these mergers, known "jet masers" (NGC 1052 and MRK 348; Claussen et al. 1998; Peck et al. 2003), and LINERs (NGC 3079, NGC 4258, NGC 6240, NGC 1052, UGC 5101) are omitted from the sample, then we obtain a slope of $m=0.5 \pm 0.1$ and a Spearman correlation coefficient of $\rho=0.5 \pm 0.1$, the latter with a significance level of $0.03 \pm 0.04$ (Table 5). To test for the robustness of this strongest correlation, we removed the data points with lowest and highest maser luminosity and obtained $m=0.5 \pm 0.1$ and $\rho=0.4 \pm 0.1$, the latter with a significance level of $0.1 \pm 0.1$ (Table 5).

The appearance of a correlation may be surprising given large uncertainties in X-ray and maser luminosities. Both are time variable, and it is easy to imagine some independence in that variability. As well, measured X-ray luminosities for heavily absorbed sources depend sensitively on the details of instrumentation and subsequent data modelling, although we have accounted for this to some extent with the adopted uncertainties. Moreover, isotropic maser luminosities should be regarded with some caution because maser beam angles are often poorly constrained and may be quite small due to source geometry and propagation effects (e.g., Deguchi \& Watson 1989; Kartje, Königl, \& Elitzur 1999). For instance, the beam angle for NGC 4258 is on the order of $7^{\circ}$ (Miyoshi et al. 1995) and, as a result, the actual luminosity is a factor of $4 \pi / \Omega=4 \pi /\left(2 \pi \times 7^{\circ}\right) \sim 16$ smaller than isotropic. Accretion disk warping may also bias maser brightness, as beamed radiation may be directed away from the observer. However, one might argue conversely based on the putative correlation that beam angles among water maser systems are relatively similar and variability is a second order effect on average. Furthermore, since the estimated power law index of $0.5 \pm 0.1$ is consistent with the value (0.5) predicted by the Neufeld \& Maloney (1995) model notwithstanding the large uncertainties that enter through the Eddington ratio, bolometric correction, viscosity, and accretion efficiency - one might venture to propose that these parameters are similar among sources, at least to within factors of a few.

The full sample presented here is inhomogeneous in that it contains AGN that are Compton-thin and Compton-thick, as well as water maser sources for which there is evidence of an origin in accretion disk material and those for which available data do not enable any particular physical classification. We have computed $m$ and $\rho$ for various sub-samples and find ranges of $0.3-0.6$ and $0.1-0.5$, respectively (Table 5). The correlation coefficient is largest $(0.5 \pm 0.1)$ and most statistically significant $(0.03 \pm 0.04)$ for the sample absent LINERs, multiple-nuclei systems, and "jet masers." No correlation is apparent for highvelocity systems due to the small sub-sample size. We have also considered Compton-thick and Compton-thin sub-samples. Since a minimum $\mathrm{H}_{2}$ density of $\sim 10^{8} \mathrm{~cm}^{-3}$ is required by the presence of water maser emission (e.g., Desch et al. 1998), the column within a disk becomes 
Compton-thick for lengths greater than $\sim 0.002 \mathrm{pc}$ and, consequently, it has been suggested that the pc-scale accretion disks that host the maser emission also provide the Compton obscuration (Greenhill et al. 2003; Herrnstein et al. 2005; Fruscione et al. 2005). We note however that there is no theoretical reason why maser emission should not originate in a Compton-thin environment. Investigation into properties of astrophysical gas by Maloney, Hollenbach, \& Tielens (1996) suggests that fractional water abundances of $10^{-6}-10^{-4}$ and temperatures of $250-1000 \mathrm{~K}$ - both conditions necessary for maser action - are possible for column densities of $\sim 10^{23} \mathrm{~cm}^{-2}$ (their Fig. 10). Assuming that the edge-on pc-scale accretion disks also provide the line-of-sight X-ray obscuration, the fact that Compton-thin environments are conducive to maser action is also supported by an empirical observation that approximately 33\% of the known nuclear masers lie in Compton-thin systems (Table 4) and that several of these are also high-velocity systems (such as NGC 4388, NGC 4258, and NGC 5728). If pc-scale accretion disks indeed provide the line-of-sight obscuring column density, factors such as the disk inclination, warping, and filling factor might affect the X-ray absorption column and it is thus possible that the intrinsic relationship between water maser and X-ray luminosities is different for Compton-thick and -thin sources. However, the two sub-samples are too small to assess any difference (Table 5).

\section{Conclusion}

In a survey with the GBT of 56 AGN with $10000 \mathrm{~km} \mathrm{~s}^{-1}<v_{\text {sys }}<30000 \mathrm{~km} \mathrm{~s}^{-1}$, we have detected five new water maser sources. The spectra of three sources display the characteristic spectral signature of emission from an edge-on accretion disk. For the 30 nuclear water masers with available hard X-ray data, we have found evidence of a possible relationship between unabsorbed X-ray luminosity $(2-10 \mathrm{keV})$ and total isotropic water maser luminosity. The power law index of $0.5 \pm 0.1$ is consistent with the Neufeld \& Maloney (1995) model in which $\mathrm{X}$-ray irradiation of molecular accretion disk gas by a central engine excites maser emission that is most intense in the disk plane. The appearance of a correlation may be surprising considering the large uncertainties in X-ray and maser luminosities, at least in part due to source variability and assumption of isotropic emission of maser radiation. However, one might interpret the correlation as indirectly supporting the proposition that Eddington ratios, bolometric corrections, viscosities, accretion efficiencies, and maser beaming angles are similar among water maser systems, and variability is a second order effect on average. Evaluation of the putative correlation would be greatly helped by an increase in the number of known maser systems and reduction in measurement and systematic uncertainties in the luminosity data. This will require measurement of X-ray spectra above $10 \mathrm{keV}$, new radio surveys of AGN (in search of maser emission), and VLBI mapping to quantify accretion disk 
structure. If verified and strengthened, the proposed relation between luminosities would be valuable in the modelling of maser excitation, identification of maser-rich samples of AGN using hard X-ray sky surveys, and perhaps modelling of the hard X-ray background in the era of the Square Kilometer Array, which will enable detection of many thousands of maser galaxies (Morganti et al. 2004).

\section{Acknowledgements}

We thank J. Braatz, M. Elvis, R. Narayan, M. Reid, and B. Wilkes for helpful discussions, C. Bignell for flexibility in GBT scheduling, and R. Maddalena for providing us with the $1.3 \mathrm{~cm}$ gain curve. We thank J. Braatz as well for help in set-up and observing. This research has made extensive use of the NASA/IPAC Extragalactic Database (NED) which is operated by the Jet Propulsion Laboratory (JPL), California Institute of Technology, under contract with NASA. This work was supported by GBT student support program, grants GSSP0040005 and GSSP004-0011.

\section{REFERENCES}

Akylas, A., Georgantopoulos, I., \& Comastri, A. 2001, MNRAS, 324, 521

Akylas, A., Georgantopoulos, I., Griffiths, R. G., Papadakis, I. E., Mastichiadis, A., Warwick, R. S., Nandra, K., \& Smith, D. A. 2002, MNRAS, 332, L23

Antonucci, R. 1993, ARA\&A, 31, 473

Armus, L., et al. 2004, ApJS, 154, 178

Ballo, L., Braito, V., Della Ceca, R., Maraschi, L., Tavecchio, F., \& Dadina, M. 2004, ApJ, 600,634

Barvainis, R., \& Antonucci, R. 2005, ApJ, 628, L89

Bassani, L., Dadina, M., Maiolino, R., Salvati, M., Risaliti, G., della Ceca, R., Matt, G., \& Zamorani, G. 1999, ApJS, 121, 473

Bianchi, S., Balestra, I., Matt, G., Guainazzi, M., \& Perola, G. C. 2003, A\&A, 402, 141

Braatz, J., Greenhill, L., Moran, J., Wilson, A., \& Herrnstein, J. 1997a, BAAS, 29, 1374 
Braatz, J. A., Henkel, C., Greenhill, L. J., Moran, J. M., \& Wilson, A. S. 2004, ApJ, 617, L29

Braatz, J. A., Wilson, A. S., \& Henkel, C. 1996, ApJS, 106, 51

-. 1997b, ApJS, 110, 321

Cappi, M., et al. 1999, A\&A, 344, 857

Claussen, M. J., Diamond, P. J., Braatz, J. A., Wilson, A. S., \& Henkel, C. 1998, ApJ, 500, L129

Colbert, E. J. M., Weaver, K. A., Krolik, J. H., Mulchaey, J. S., \& Mushotzky, R. F. 2002, ApJ, 581, 182

Comastri, A. 2004, Compton-Thick AGN: The Dark Side of the X-Ray Background (ASSL Vol. 308: Supermassive Black Holes in the Distant Universe), 245

Condon, J. J. 2003, GBT PTCS Project Notes, 26

Deguchi, S., \& Watson, W. D. 1989, ApJ, 340, L17

Della Ceca, R., et al. 2002, ApJ, 581, L9

Desch, S. J., Wallin, B. K., \& Watson, W. D. 1998, ApJ, 496, 775

Done, C., Madejski, G. M., Życki, P. T., \& Greenhill, L. J. 2003, ApJ, 588, 763

Elvis, M., Risaliti, G., Nicastro, F., Miller, J. M., Fiore, F., \& Puccetti, S. 2004, ApJ, 615, L25

Elvis, M., Risaliti, G., \& Zamorani, G. 2002, ApJ, 565, L75

Fabian, A. C., \& Iwasawa, K. 1999, MNRAS, 303, L34

Franceschini, A., et al. 2005, AJ, 129, 2074

Frank, J., King, A., \& Raine, D. J. 2002, Accretion Power in Astrophysics: Third Edition (Accretion Power in Astrophysics: Third Edition, by Juhan Frank, Andrew King, and Derek J. Raine. Cambridge University Press, 2002, 398 pp.)

Fruscione, A., Greenhill, L. J., Filippenko, A. V., Moran, J. M., Herrnstein, J. R., \& Galle, E. 2005, ApJ, 624, 103 
Fukazawa, Y., Iyomoto, N., Kubota, A., Matsumoto, Y., \& Makishima, K. 2001, A\&A, 374, 73

Gallimore, J. F., Henkel, C., Baum, S. A., Glass, I. S., Claussen, M. J., Prieto, M. A., \& Von Kap-herr, A. 2001, ApJ, 556, 694

Georgantopoulos, I., Papadakis, I., Warwick, R. S., Smith, D. A., Stewart, G. C., \& Griffiths, R. G. 1999, MNRAS, 307, 815

Georgantopoulos, I., \& Papadakis, I. E. 2001, MNRAS, 322, 218

Gimeno, G. N., Díaz, R. J., \& Carranza, G. J. 2004, AJ, 128, 62

Greenhill, L. J., \& Gwinn, C. R. 1997, Ap\&SS, 248, 261

Greenhill, L. J., Kondratko, P. T., Lovell, J. E. J., Kuiper, T. B. H., Moran, J. M., Jauncey, D. L., \& Baines, G. P. 2003, ApJ, 582, L11

Greenhill, L. J., Moran, J. M., \& Herrnstein, J. R. 1997, ApJ, 481, L23

Greenhill, L. J., et al. 2003, ApJ, 590, 162

Guainazzi, M. 2002, MNRAS, 329, L13

Guainazzi, M., \& Antonelli, L. A. 1999, MNRAS, 304, L15

Guainazzi, M., Fabian, A. C., Iwasawa, K., Matt, G., \& Fiore, F. 2005, MNRAS, 356, 295

Guainazzi, M., Matt, G., Brandt, W. N., Antonelli, L. A., Barr, P., \& Bassani, L. 2000a, A\&A, 356, 463

Guainazzi, M., Oosterbroek, T., Antonelli, L. A., \& Matt, G. 2000b, A\&A, 364, L80

Guainazzi, M., Rodriguez-Pascual, P., Fabian, A. C., Iwasawa, K., \& Matt, G. 2004, MNRAS, 355, 297

Guainazzi, M., et al. 1999, MNRAS, 310, 10

Hagiwara, Y., Diamond, P. J., \& Miyoshi, M. 2003a, A\&A, 400, 457

Hagiwara, Y., Diamond, P. J., Miyoshi, M., Rovilos, E., \& Baan, W. 2003b, MNRAS, 344, L53

Hagiwara, Y., Henkel, C., Menten, K. M., \& Nakai, N. 2001, ApJ, 560, L37 
Henkel, C., Peck, A. B., Tarchi, A., Nagar, N. M., Braatz, J. A., Castangia, P., \& Moscadelli, L. 2005, A\&A, 436, 75

Henkel, C., Wang, Y. P., Falcke, H., Wilson, A. S., \& Braatz, J. A. 1998, A\&A, 335, 463

Herrnstein, J. R., Moran, J. M., Greenhill, L. J., \& Trotter, A. S. 2005, ApJ, 629, 719

Herrnstein, J. R., et al. 1999, Nature, 400, 539

Ho, L. C. 1999a, Advances in Space Research, 23, 813

-. 1999b, ApJ, 516, 672

Ho, L. C., Filippenko, A. V., \& Sargent, W. L. W. 1997, ApJ, 487, 568

-. 2003, ApJ, 583, 159

Ikebe, Y., Leighly, K., Tanaka, Y., Nakagawa, T., Terashima, Y., \& Komossa, S. 2000, MNRAS, 316, 433

Ishihara, Y., Nakai, N., Iyomoto, N., Makishima, K., Diamond, P., \& Hall, P. 2001, PASJ, 53, 215

Iwasawa, K., Maloney, P. R., \& Fabian, A. C. 2002, MNRAS, 336, L71

Iyomoto, N., Fukazawa, Y., Nakai, N., \& Ishihara, Y. 2001, ApJ, 561, L69

Kadler, M., Kerp, J., Ros, E., Falcke, H., Pogge, R. W., \& Zensus, J. A. 2004, A\&A, 420, 467

Kartje, J. F., Königl, A., \& Elitzur, M. 1999, ApJ, 513, 180

Kondratko, P. T., Greenhill, L. J., \& Moran, J. M. 2005, ApJ, 618, 618

Kondratko, P. T., et al. 2006, ApJ, 638, 100

Kraft, R. P., Hardcastle, M. J., Worrall, D. M., \& Murray, S. S. 2005, ApJ, 622, 149

Kuraszkiewicz, J. K., et al. 2003, ApJ, 590, 128

Lamer, G., Uttley, P., \& McHardy, I. M. 2000, MNRAS, 319, 949

Lawrence, A., \& Elvis, M. 1982, ApJ, 256, 410

Leighly, K. M., Halpern, J. P., Awaki, H., Cappi, M., Ueno, S., \& Siebert, J. 1999, ApJ, 522,209 
Levenson, N. A., Weaver, K. A., \& Heckman, T. M. 2001, ApJS, 133, 269

Lira, P., Ward, M. J., Zezas, A., \& Murray, S. S. 2002, MNRAS, 333, 709

Lutz, D., Maiolino, R., Spoon, H. W. W., \& Moorwood, A. F. M. 2004, A\&A, 418, 465

Maddox, L. A., Leighly, K. M., Nava, A., Matsumoto, C., \& Grupe, D. 2002, BAAS, 34, 1183

Madejski, G., Done, C., Życki, P. T., \& Greenhill, L. 2006, ApJ, 636, 75

Maiolino, R., Salvati, M., Bassani, L., Dadina, M., della Ceca, R., Matt, G., Risaliti, G., \& Zamorani, G. 1998, A\&A, 338, 781

Makishima, K., Ohashi, T., Kondo, H., Palumbo, G. G. C., \& Trinchieri, G. 1990, ApJ, 365, 159

Maloney, P. R., Hollenbach, D. J., \& Tielens, A. G. G. M. 1996, ApJ, 466, 561

Matsumoto, C., Nava, A., Maddox, L. A., Leighly, K. M., Grupe, D., Awaki, H., \& Ueno, S. 2004, ApJ, 617, 930

Matt, G., Fabian, A. C., Guainazzi, M., Iwasawa, K., Bassani, L., \& Malaguti, G. 2000, MNRAS, 318, 173

Matt, G., Guainazzi, M., Perola, G. C., Fiore, F., Nicastro, F., Cappi, M., \& Piro, L. 2001, A\&A, 377, L31

Matt, G., et al. 1999, A\&A, 341, L39

McHardy, I. M., Papadakis, I. E., Uttley, P., Page, M. J., \& Mason, K. O. 2004, MNRAS, 348,783

Miyoshi, M., Moran, J., Herrnstein, J., Greenhill, L., Nakai, N., Diamond, P., \& Inoue, M. 1995, Nature, 373, 127

Modjaz, M., Moran, J. M., Kondratko, P. T., \& Greenhill, L. J. 2005, ApJ, 626, 104

Morganti, R., Greenhill, L. J., Peck, A. B., Jones, D. L., \& Henkel, C. 2004, New Astronomy Review, 48, 1195

Nandra, K., \& Pounds, K. A. 1994, MNRAS, 268, 405

Neufeld, D. A., \& Maloney, P. R. 1995, ApJ, 447, L17 
Neufeld, D. A., Maloney, P. R., \& Conger, S. 1994, ApJ, 436, L127

Ohno, M., Fukazawa, Y., \& Iyomoto, N. 2004, PASJ, 56, 425

O’Neill, P. M., Nandra, K., Papadakis, I. E., \& Turner, T. J. 2005, MNRAS, 358, 1405

Padovani, P. 1989, A\&A, 209, 27

Peck, A. B., Henkel, C., Ulvestad, J. S., Brunthaler, A., Falcke, H., Elitzur, M., Menten, K. M., \& Gallimore, J. F. 2003, ApJ, 590, 149

Perola, G. C., Matt, G., Cappi, M., Fiore, F., Guainazzi, M., Maraschi, L., Petrucci, P. O., \& Piro, L. 2002, A\&A, 389, 802

Pounds, K. A., Reeves, J. N., King, A. R., \& Page, K. L. 2004, MNRAS, 350, 10

Ptak, A., Heckman, T., Levenson, N. A., Weaver, K., \& Strickland, D. 2003, ApJ, 592, 782

Risaliti, G. 2002, A\&A, 386, 379

Risaliti, G., Gilli, R., Maiolino, R., \& Salvati, M. 2000, A\&A, 357, 13

Risaliti, G., Maiolino, R., \& Salvati, M. 1999, ApJ, 522, 157

Sako, M., Kahn, S. M., Paerels, F., \& Liedahl, D. A. 2000, ApJ, 543, L115

Salvati, M., Bassani, L., della Ceca, R., Maiolino, R., Matt, G., \& Zamorani, G. 1997, A\&A, 323, L1

Satyapal, S., Dudik, R. P., O’Halloran, B., \& Gliozzi, M. 2005, ApJ, 633, 86

Satyapal, S., Sambruna, R. M., \& Dudik, R. P. 2004, A\&A, 414, 825

Shakura, N. I., \& Sunyaev, R. A. 1973, A\&A, 24, 337

Smith, D. A., \& Done, C. 1996, MNRAS, 280, 355

Smith, D. A., \& Wilson, A. S. 2001, ApJ, 557, 180

Tarchi, A., Henkel, C., Chiaberge, M., \& Menten, K. M. 2003, A\&A, 407, L33

Terashima, Y., Ho, L. C., \& Ptak, A. F. 2000, ApJ, 539, 161

Terashima, Y., Iyomoto, N., Ho, L. C., \& Ptak, A. F. 2002, ApJS, 139, 1 
Trotter, A. S., Greenhill, L. J., Moran, J. M., Reid, M. J., Irwin, J. A., \& Lo, K. 1998, ApJ, 495,740

Ueda, Y., Ishisaki, Y., Takahashi, T., Makishima, K., \& Ohashi, T. 2001, ApJS, 133, 1

Uttley, P., Fruscione, A., McHardy, I., \& Lamer, G. 2003, ApJ, 595, 656

Uttley, P., McHardy, I. M., Papadakis, I. E., Guainazzi, M., \& Fruscione, A. 1999, MNRAS, 307, L6

Uttley, P., Taylor, R. D., McHardy, I. M., Page, M. J., Mason, K. O., Lamer, G., \& Fruscione, A. 2004, MNRAS, 347, 1345

Vignati, P., et al. 1999, A\&A, 349, L57

Wall, J. V., \& Jenkins, C. R. 2003, Practical Statistics for Astronomers (Princeton Series in Astrophysics)

Wandel, A., Peterson, B. M., \& Malkan, M. A. 1999, ApJ, 526, 579

Wang, J., Heckman, T. M., Weaver, K. A., \& Armus, L. 1997, ApJ, 474, 659

Wang, T., Mihara, T., Otani, C., Matsuoka, M., \& Awaki, H. 1999, ApJ, 515, 567

Warwick, R. S., Koyama, K., Inoue, H., Takano, S., Awaki, H., \& Hoshi, R. 1989, PASJ, 41, 739

Weaver, K. A., Wilson, A. S., Henkel, C., \& Braatz, J. A. 1999, ApJ, 520, 130

Wilkes, B. J., Mathur, S., Fiore, F., Antonelli, A., \& Nicastro, F. 2001, ApJ, 549, 248

Wilson, A. S., et al. 1998, ApJ, 505, 587

Zezas, A., Ward, M. J., \& Murray, S. S. 2003, ApJ, 594, L31

Zhang, J. S., Henkel, C., Kadler, M., Greenhill, L. J., Nagar, N., Wilson, A. S., \& Braatz, J. A. 2006, A\&A, 450, 933

This preprint was prepared with the AAS LATEX macros v5.2. 
Table 1. Galaxies Surveyed for Water Maser Emission with the Green Bank Telescope. [Refer to the source file for the complete version of this table.]

\begin{tabular}{llcccccc}
\hline \hline \multicolumn{1}{c}{ Galaxy } & Type $^{(a)}$ & $\begin{array}{c}\alpha_{2000} \\
(\mathrm{hh} \mathrm{mm} \mathrm{ss})\end{array}$ & $\begin{array}{c}\delta_{2000}{ }^{(a)} \\
(\mathrm{dd} \mathrm{mm} \mathrm{ss})\end{array}$ & $\begin{array}{c}\mathrm{v}_{\text {sys }}{ }^{(a)} \\
\left(\mathrm{km} \mathrm{s}^{-1}\right)\end{array}$ & Date & $\begin{array}{c}T_{\text {sys }}{ }^{(b)} \\
(\mathrm{K})\end{array}$ & $\begin{array}{c}1 \sigma^{(c)} \\
(\mathrm{mJy})\end{array}$ \\
\hline UM213 & LINER & 001217.83 & -000610.6 & 12149 & $11-10-2003$ & 30 & 4.6 \\
MRK0948 & Sy2 & 002814.34 & +070745.4 & 12022 & $11-08-2003$ & 42 & 4.8 \\
UM254 & Sy2 & 003134.27 & -020916.8 & 13311 & $11-10-2003$ & 32 & 5.9 \\
FGC0061 & Sy2 & 003443.51 & -000226.7 & 12610 & $11-10-2003$ & 28 & 3.7 \\
LEDA093200 & Sy2 & 004135.07 & -092152.2 & 14234 & $11-10-2003$ & 29 & 4.0 \\
\hline
\end{tabular}

${ }^{(a)}$ Type, position, and heliocentric optical systemic velocity obtained from the NED at the outset of the survey in 2002.

${ }^{(b)}$ Average system temperature.

${ }^{(c)} \mathrm{Rms}$ noise in a $24.4 \mathrm{kHz}$ spectral channel, corrected for atmospheric opacity (typically from 0.02 to 0.07 ) and for the dependence of antenna gain on elevation. 
Table 2. Newly Discovered Nuclear Water Maser Sources.

\begin{tabular}{|c|c|c|c|c|c|c|c|c|c|}
\hline $\begin{array}{l}\text { Galaxy } \\
\text { Name }\end{array}$ & Type $^{(a)}$ & $\begin{array}{c}\alpha_{2000}{ }^{(b)} \\
(\mathrm{hh} \mathrm{mm} \mathrm{ss})\end{array}$ & $\begin{array}{c}\delta_{2000}{ }^{(b)} \\
(\operatorname{dd} \mathrm{mm} \mathrm{ss})\end{array}$ & $\begin{array}{r}\mathrm{v}_{\text {sys }}{ }^{(c)} \\
\left(\mathrm{km} \mathrm{s}^{-1}\right)\end{array}$ & Date & $\begin{array}{l}T_{\text {sys }}{ }^{(d)} \\
(\mathrm{K})\end{array}$ & $\begin{array}{c}1 \sigma^{(e)} \\
(\mathrm{mJy})\end{array}$ & $\begin{array}{c}\Delta \nu^{(f)} \\
(\mathrm{kHz})\end{array}$ & $\begin{array}{c}\mathrm{BW}^{(g)} \\
(\mathrm{MHz})\end{array}$ \\
\hline IRAS $03355+0104$ & Sy2 & $\begin{array}{lll}03 & 38 & 10.38 \\
03 & 38 & 10.38\end{array}$ & $\begin{array}{l}+011418.2 \\
+011418.3\end{array}$ & 11926 & 11-08-2003 & 38 & 3.2 & 195 & 12.5 \\
\hline 2MASX J08362280+3327383 & Sy2 & $\begin{array}{lll}08 & 36 & 22.80 \\
08 & 36 & 22.80\end{array}$ & $\begin{array}{l}+332738.6 \\
+332738.8\end{array}$ & 14810 & 01-15-2005 & 25 & 1.5 & 97.7 & 6.25 \\
\hline SBS $0927+493$ & LINER & $\begin{array}{l}093106.76 \\
093106.77\end{array}$ & $\begin{array}{l}+490447.5 \\
+490447.2\end{array}$ & 10167 & 02-01-2006 & 37 & 2.3 & 195 & 12.5 \\
\hline UGC 09618 NED02 & LINER & 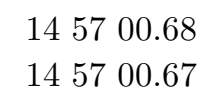 & $\begin{array}{l}+243702.7 \\
+243702.9\end{array}$ & 10094 & $01-15-2005$ & 26 & 1.4 & 195 & 12.5 \\
\hline NGC 6264 & Sy2 & $\begin{array}{lll}16 & 57 & 16.12 \\
16 & 57 & 16.13\end{array}$ & $\begin{array}{l}+275058.7 \\
+275058.6\end{array}$ & 10177 & $01-27-2005$ & 23 & 1.3 & 195 & 12.5 \\
\hline
\end{tabular}

${ }^{(a)}$ Activity type from the NED.

(b) First line: optical positions from the NED with uncertainties of $\pm 0 .{ }^{\prime \prime} 5$ (SBS 0927+493) or $\pm 11^{\prime \prime} 3$. Second line: maser positions measured with a VLA snapshot, providing typical uncertainties of $\pm 0{ }^{\prime \prime} 3$. To establish the magnitude of systematic uncertainties in position, we imaged disjoint segments of the VLA data and confirmed that they yield consistent maser positions (that is, within 0 .' 3 ).

${ }^{(c)}$ Optical heliocentric systemic velocity from the NED.

${ }^{(d)}$ Average system temperature.

${ }^{(e)} \mathrm{Rms}$ noise in a $24.4 \mathrm{kHz}$ spectral channel, corrected for atmospheric opacity (typically from 0.02 to 0.07 ) and for the dependence of antenna gain on elevation.

(f) VLA channel width.

${ }^{(g)}$ VLA bandwidth; the VLA band was in each case centered on the strongest maser line. 
Table 3. Known Water Maser Sources Reobserved with the Green Bank Telescope.

\begin{tabular}{|c|c|c|c|c|c|c|c|}
\hline Galaxy & Type $^{(a)}$ & $\begin{array}{c}\alpha_{2000}{ }^{(b)} \\
(\mathrm{hh} \mathrm{mm} \mathrm{ss})\end{array}$ & $\begin{array}{c}\delta_{2000}{ }^{(b)} \\
(\mathrm{dd} \mathrm{mm} \mathrm{ss})\end{array}$ & $\begin{array}{r}\mathrm{v}_{\text {sys }}{ }^{(c)} \\
\left(\mathrm{km} \mathrm{s}^{-1}\right)\end{array}$ & Date & $\begin{array}{l}T_{\text {sys }}{ }^{(d)} \\
(\mathrm{K})\end{array}$ & $\begin{array}{r}1 \sigma^{(e)} \\
(\mathrm{mJy})\end{array}$ \\
\hline IC 0184 & Sy2 & 015951.23 & -065025.4 & 5382 & 07-03-2005 & 71 & 8.9 \\
\hline VII ZW 073 & Sy2 & 063025.54 & +634041.3 & 12391 & 01-01-2005 & 35 & 2.6 \\
\hline MRK 0034 & Sy2 & 103408.592 & +600152.01 & 15140 & 01-18-2005 & 21 & 0.98 \\
\hline NGC 3393 & Sy2 & 104823.45 & -250943.6 & 3750 & $01-15-2005$ & 37 & 1.9 \\
\hline NGC 5495 & Sy2 & 141223.35 & -270629.2 & 6737 & 02-09-2006 & 40 & 2.4 \\
\hline AM 2158-380 NED02 & Sy2 & 220117.10 & -374623.0 & 9983 & 07-03-2005 & 99 & 22 \\
\hline
\end{tabular}

${ }^{(a)}$ Activity type from the NED.

(b) Positions from Kondratko et al. $2006\left(\sigma=00^{\prime \prime} 3\right)$ except for MRK 0034, which is from the NED $\left(\sigma=0{ }^{\prime \prime} 3\right)$.

${ }^{(c)}$ Optical heliocentric systemic velocity from the NED.

${ }^{(d)}$ Average system temperature.

${ }^{(e)} \mathrm{Rms}$ noise in a $24.4 \mathrm{kHz}$ spectral channel, corrected for atmospheric opacity (typically from 0.02 to 0.07 ) and for the dependence of antenna gain on elevation. 
Table 4. Water Maser and Unabsorbed 2-10 keV Luminosities.

\begin{tabular}{|c|c|c|c|c|c|c|c|}
\hline \multirow{2}{*}{$\begin{array}{r}\text { Galaxy } \\
\text { NGC } 1068\end{array}$} & \multirow{2}{*}{$\begin{array}{r}\begin{array}{r}\mathrm{D}^{(a)} \\
(\mathrm{Mpc})\end{array} \\
14\end{array}$} & \multirow{2}{*}{$\frac{\begin{array}{c}\log L_{\mathrm{H}_{2} \mathrm{O}^{(b)}} \\
\left(L_{\odot}\right)\end{array}}{1.7}$} & \multirow{2}{*}{$\begin{array}{c}\frac{\log L_{2-10}(c)}{\left(L_{\odot}\right)} \\
>>9.6\end{array}$} & \multirow{2}{*}{$\begin{array}{l}\text { Telescopes }^{(d)} \\
\text { ABR }\end{array}$} & \multirow{2}{*}{$\operatorname{Ref}^{(e)}$} & \multicolumn{2}{|c|}{ Type $^{(f)} \operatorname{Ref}^{(g)}$} \\
\hline & & & & & & $\mathrm{DC}$ & $\mathrm{A}$ \\
\hline Circinus & 4.0 & 1.3 & $7.5-8.6$ & $\mathrm{~B}$ & $2-4$ & $\mathrm{DC}$ & $\mathrm{B}$ \\
\hline NGC 4945 & 4.0 & 1.7 & $9.1-9.6$ & $\mathrm{BCR}$ & 5,6 & $\mathrm{DC}$ & $\mathrm{C}$ \\
\hline M 51 & 10 & -0.2 & $7.7-8.4$ & $\mathrm{BG}$ & 7,8 & $\mathrm{X} \mathrm{C}$ & $\mathrm{D}$ \\
\hline NGC 3079 & 16 & 2.5 & $8.4-9.4$ & $\mathrm{~B}$ & 9 & $\mathrm{DC}$ & $\mathrm{E}$ \\
\hline NGC 1386 & 17 & 2.1 & $8.4-9.0$ & $\mathrm{BCX}$ & 10,11 & $\mathrm{DC}$ & $\mathrm{F}$ \\
\hline NGC 3393 & 50 & 2.4 & $8.5-10.2$ & $\mathrm{BX}$ & $10,12,13$ & $\mathrm{~d} C$ & $\mathrm{G}$ \\
\hline IC 2560 & 35 & 2.0 & 8.3-9.2 & $\mathrm{AC}$ & 14,15 & $\mathrm{DC}$ & $\mathrm{H}$ \\
\hline NGC 4051 & 10 & 0.3 & $7.6-8.5$ & CBRX & $16-20$ & $\mathrm{X} \mathrm{c}$ & $\mathrm{I}$ \\
\hline NGC 4388 & 34 & 1.1 & $8.7-9.5$ & ABCRSX & 21 & $\mathrm{~d} \mathrm{c}$ & $\mathrm{J}$ \\
\hline 3C 403 & 235 & 3.3 & $9.6-10.3$ & $\mathrm{C}$ & 22 & $\mathrm{Xc}$ & $\mathrm{K}$ \\
\hline NGC 4258 & 7.2 & 1.9 & $7.0-7.7$ & $\mathrm{ABCX}$ & 23 & $\mathrm{D} \mathrm{c}$ & $\mathrm{L}$ \\
\hline NGC 6240 & 98 & 1.6 & $9.8-10.8$ & ABR & 24,25 & $\mathrm{X} \mathrm{C}$ & M \\
\hline MRK 266 & 110 & 1.5 & $9.3-10.3$ & $\mathrm{~B}$ & 26 & $\mathrm{X} \mathrm{C}$ & $\mathrm{J}$ \\
\hline MRK 3 & 54 & 1.0 & $9.6-10.4$ & BCGR & $27-32$ & $\mathrm{X} \mathrm{C}$ & $\mathrm{J}$ \\
\hline NGC 5643 & 16 & 1.3 & $7.6-9.0$ & BX & 13,33 & $\mathrm{X} \mathrm{C} ?$ & $\mathrm{G}$ \\
\hline NGC 5347 & 31 & 1.5 & $8.1-9.1$ & $\mathrm{~A}$ & 14 & $\mathrm{X} \mathrm{C}$ & $\mathrm{N}$ \\
\hline MRK 1066 & 48 & 1.5 & $9.0-9.2$ & $\mathrm{~A}$ & 34 & $\mathrm{X} \mathrm{C}$ & $\mathrm{J}$ \\
\hline ESO 103-G 035 & 53 & 2.6 & $9.1-9.9$ & ABEGR & $35-38$ & $\mathrm{X} \mathrm{c}$ & $\mathrm{N}$ \\
\hline NGC 6300 & 15 & 0.34 & $8.1-8.5$ & $\mathrm{BRX}$ & $35,39-41,67$ & $\mathrm{Xc}$ & $\mathrm{G}$ \\
\hline NGC 5506 & 25 & 1.7 & 8.9-9.4 & ABCGRX & $35,42-48$ & $\mathrm{X} \mathrm{c}$ & $\mathrm{N}$ \\
\hline NGC 1052 & 17 & 2.1 & $7.5-8.0$ & $\mathrm{ABC}$ & $49-55$ & $\mathrm{~J} \mathrm{c}$ & $\mathrm{O}$ \\
\hline MRK 348 & 62 & 2.6 & 8.9-10.4 & $\mathrm{AG}$ & $32,56,57$ & $\mathrm{~J} \mathrm{c}$ & $\mathrm{P}$ \\
\hline MRK 1210 & 54 & 1.9 & $9.3-10.3$ & $\mathrm{AB}$ & 58,59 & $\mathrm{~d} C ?$ & $\mathrm{~T}$ \\
\hline NGC 2639 & 44 & 1.4 & $7.0-8.1$ & $\mathrm{~A}$ & $52,59-61$ & $\mathrm{Xc}$ & $\mathrm{N}$ \\
\hline UGC 5101 & 157 & 3.2 & $8.8-9.8$ & $\mathrm{C}$ & 62 & $\mathrm{X} \mathrm{C}$ & $\mathrm{R}$ \\
\hline NGC 2273 & 25 & 0.8 & $8.1-9.3$ & BX & 10,13 & $\mathrm{X} \mathrm{C}$ & $\mathrm{R}$ \\
\hline NGC 2782 & 34 & 1.1 & $7.6-9.1$ & $\mathrm{C}$ & 63 & $\mathrm{X} \mathrm{C}$ & $\mathrm{J}$ \\
\hline ARP 299 & 42 & 2.1 & $8.2-9.4$ & $\mathrm{BCX}$ & 64-66 & $\mathrm{X} \mathrm{C}$ & $\mathrm{S}$ \\
\hline
\end{tabular}


Table 4-Continued

\begin{tabular}{|c|c|c|c|c|c|c|}
\hline Galaxy & $\begin{array}{r}\mathrm{D}^{(a)} \\
(\mathrm{Mpc})\end{array}$ & $\begin{array}{c}\log L_{\mathrm{H}_{2} \mathrm{O}}{ }^{(b)} \\
\left(L_{\odot}\right)\end{array}$ & $\begin{array}{c}\log L_{2-10^{(c)}} \\
\left(L_{\odot}\right)\end{array}$ & Telescopes $^{(d)}$ & $\operatorname{Ref}^{(e)}$ & Type $^{(f)} \operatorname{Ref}^{(g)}$ \\
\hline NGC 5728 & 37 & 1.9 & $8.7-10.2$ & $\mathrm{C}$ & 63 & $\mathrm{~d} C ?$ \\
\hline
\end{tabular}

${ }^{(a)}$ Distances adopted from Henkel et al. (2005).

(b) Total isotropic water maser luminosities from Henkel et al. (2005) except for NGC 3393, NGC 5643, NGC 6300, NGC 1068 (Kondratko et al. 2006), NGC 3079 (Kondratko et al. 2005), UGC 5101, and NGC 2273 (Zhang et al. 2006).

(c) Unabsorbed 2-10 keV luminosity. In cases where this is not described in the literature, the unabsorbed $2-10 \mathrm{keV}$ luminosity has been inferred from other reported parameters (flux, photon index, and the neutral hydrogen column density) using WebPIMMS. The ranges quoted here reflect results from multiple observations, alternative source models, and/or the uncertainty in the reflection efficiency (in case of Compton-thick sources only; see note $\dagger$ below). To account for source variability in case of $3 \mathrm{C} 403$, where multiple observations and alternative models were not available, we adopted the fractional uncertainty averaged over all Compton-thin systems in our sample. Only observations sensitive to energies above $10 \mathrm{keV}$ were considered for Compton-thick sources, with the exception of NGC 1386, IC 2560, NGC 5347, MRK 1066, UGC 5101, NGC 2782, and NGC 5728 for which hard X-ray data were not available.

${ }^{(d)} \mathrm{X}$-ray telescopes used in luminosity measurements: $\mathrm{A}=\mathrm{ASCA}, \mathrm{B}=\mathrm{BeppoSAX}, \mathrm{C}=\mathrm{CXO}, \mathrm{E}=\mathrm{EXOSAT}, \mathrm{G}=\mathrm{GINGA}, \mathrm{R}=\mathrm{RXTE}, \mathrm{S}=\mathrm{SL} 2-\mathrm{XRT}$, $\mathrm{X}=\mathrm{XMM}$.

(e) References for the 2-10 keV luminosity: [1] Colbert et al. (2002) but also see Comastri (2004) and references therein, [2] Guainazzi et al. (1999), [3] Matt et al. (1999), [4] Smith \& Wilson (2001), [5] Guainazzi et al. (2000a), [6] Done et al. (2003), [7] Fukazawa et al. (2001), [8] Makishima et al. (1990), [9] Iyomoto et al. (2001), [10] Guainazzi et al. (2005), [11] Comastri (2004), [12] Salvati et al. (1997), [13] Maiolino et al. $(1998)^{\dagger}$, [14] Risaliti et al. (1999) ${ }^{\dagger},[15]$ Iwasawa et al. (2002), [16] Pounds et al. (2004), [17] Uttley et al. (2004), [18] McHardy et al. (2004), [19] Uttley et al. (2003), [20] Uttley et al. (1999), [21] Elvis et al. (2004), [22] Kraft et al. (2005), [23] Fruscione et al. (2005), [24] Ikebe et al. (2000), [25] Vignati et al. (1999), [26] Risaliti et al. (2000) ${ }^{\dagger}$, [27] Lutz et al. (2004), [28] Matt et al. (2000), [29] Sako et al. (2000), [30] Capi et al. (1999), [31] Georgantopoulos et al. (1999), [32] Smith \& Done (1996), [33] Guainazzi et al. (2004), [34] Levenson et al. (2001), [35] Risaliti (2002), [36] Wilkes et al. (2001), [37] Akylas et al. (2001), [38] Georgantopoulos \& Papadakis (2001), [39] Maddox et al. (2002), [40] Guainazzi (2002), [41] Leighly et al. (1999), [42] O'Neill et al. (2005), [43] Bianchi et al. (2003), [44] Perola et al. (2002), [45] Matt et al. (2001), [46] Lamer et al. (2000), [47] Wang et al. (1999), [48] Nandra \& Pounds (1994), [49] Kadler et al. (2004), [50] Satyapal et al. (2004), [51] Ueda et al. (2001), [52] Terashima et al. (2000), [53] Guainazzi et al. (2000b), [54] Weaver et al. (1999), [55] Guainazzi \& Antonelli (1999), [56] Akylas et al. (2002), [57] Warwick et al. (1989), [58] Ohno et al. (2004), [59] Bassani et al. (1999) ${ }^{\dagger}$, [60] Terashima et al. (2002), [61] Wilson et al. (1998), [62] Assuming that the source is Compton-thick based on Armus et al. (2004) and using the measured flux from Ptak et al. (2003) ${ }^{\dagger}$, [63] Zhang et al. (2006) ${ }^{\dagger}$, [64] Della Ceca, R., et al. (2002), [65] Ballo et al. (2004) ${ }^{\dagger}$, [66] Zezas et al. (2003) ${ }^{\dagger}$, [67] Matsumoto et al. (2004).

$(f)$ Two letter code describing (1) maser type and (2) magnitude of X-ray absorption column. Maser type: d=high-velocity water maser system, i.e., a source where origin of emission in an edge-on disk is suggested by spectral data only; $\mathrm{D}=$ high-velocity water maser systems, where hypothesized disk origin has been reinforced by an analysis of VLBI data; J=maser source associated with a radio jet; X=non-high-velocity system. Obscuration: $\mathrm{C}=$ Compton-thick; $\mathrm{c}=$ Compton-thin. A question mark after obscuration type indicates controversy in analysis of $\mathrm{X}$-ray data reported in the literature.

(g) References for the maser type: [A] Greenhill \& Gwinn (1997), [B] Greenhill, L. J., et al. (2003), [C] Greenhill et al. (1997), [D] Hagiwara et al. (2001), [E] Kondratko et al. (2005), [F] Braatz et al. (1997a), [G] Kondratko et al. 2006, [H] Ishihara et al. (2001), [I] Hagiwara et al. (2003b), [J] Braatz et al. (2004), [K] Tarchi et al. (2003), [L] Miyoshi et al. (1995), [M] Hagiwara et al. (2003a), [N] Braatz et al. (1996), [O] Claussen et al. (1998), [P] Peck et al. (2003), [R] Zhang et al. (2006), [S] Henkel et al. (2005) [T] Kondratko et al., in preparation.

${ }^{\dagger}$ Following Comastri (2004), we assumed in case of Compton-thick models for NGC 5347, NGC 5643, NGC 3393, MRK 266, MRK 1210, UGC 5101, NGC 2273, NGC 2782, ARP 299, and NGC 5728 that the observed $2-10 \mathrm{keV}$ luminosity is $1-10 \%$ of the unabsorbed $2-10$ keV luminosity due to reflection and scattering. 
Table 5. Correlation Coefficients and Power Law Indices for $L_{2-10}$ vs. $L_{\mathrm{H}_{2} \mathrm{O}}$.

\begin{tabular}{lcccc}
\hline \hline Population & $m^{(a)}$ & $\rho^{(b)}$ & Significance & $\mathrm{N}^{(d)}$ \\
& & & & \\
\hline all systems & $0.4 \pm 0.1(0.4 \pm 0.1)$ & $0.4 \pm 0.1(0.3 \pm 0.1)$ & $7 \pm 7(20 \pm 10)$ & 29 \\
all systems $^{\dagger}$ & $0.5 \pm 0.1(0.5 \pm 0.1)$ & $0.5 \pm 0.1(0.4 \pm 0.1)$ & $3 \pm 4(10 \pm 10)$ & 20 \\
unclassified and jet masers $^{(c)}$ & $0.5 \pm 0.1(0.4 \pm 0.1)$ & $0.5 \pm 0.1(0.4 \pm 0.1)$ & $5 \pm 6(20 \pm 10)$ & 19 \\
high-velocity & $0.4 \pm 0.3(1.0 \pm 0.5)$ & $0.1 \pm 0.2(0.2 \pm 0.2)$ & $70 \pm 20(60 \pm 20)$ & 10 \\
Compton-thick & $0.3 \pm 0.1(0.2 \pm 0.2)$ & $0.3 \pm 0.1(0.1 \pm 0.1)$ & $30 \pm 20(60 \pm 20)$ & 19 \\
Compton-thin & $0.6 \pm 0.1(0.5 \pm 0.2)$ & $0.5 \pm 0.1(0.3 \pm 0.1)$ & $20 \pm 10(40 \pm 20)$ & 10 \\
\hline
\end{tabular}

${ }^{(a)}$ Index $\left(L_{2-10} \propto L_{\mathrm{H}_{2} \mathrm{O}}^{m}\right)$ obtained from a Monte Carlo simulation where the probability distribution function for each data point in Fig. 3 is modelled in linear luminosity space by a Gaussian with mean and standard deviation corresponding to the plotted symbols and the error bars in Fig. 3, respectively. The parentheses enclose the result for the same population but with the leftmost and the rightmost data points in Fig. 3 removed.

${ }^{(b)}$ Spearman correlation coefficient and uncertainty from the Monte Carlo simulation described in (a). Alternative use of a Pearson correlation coefficient changes the results negligibly but assumes in the calculation of the significance level that the two variables are normally distributed, which is most likely not the case here. Quantities in parentheses are as in (a).

(c) Two-sided significance level (expressed as a percentage), i.e., probability that a random data set of the same size would yield a larger magnitude of the correlation coefficient. Quantities in parentheses are as in (a). We have confirmed the reported significance levels with numerous $\left(\sim 10^{5}\right)$ realizations of the permutation test, whereas the null hypothesis of no correlation is assumed and the water luminosity data points are permuted among the x-ray luminosity data points (e.g., Wall \& Jenkins 2003).

${ }^{(d)}$ Number of sources. The lower limit on the X-ray luminosity of NGC 1068 was not used in the fit.

${ }^{\dagger}$ Galaxies with multiple nuclei (NGC 6240, MRK 266, MRK 1066, ARP 299), jet masers (MRK 348, NGC 1052), and LINER systems (NGC 3079, NGC 4258, NGC 6240, NGC 1052, UGC 5101) have been removed from the sample. See the text for details. 

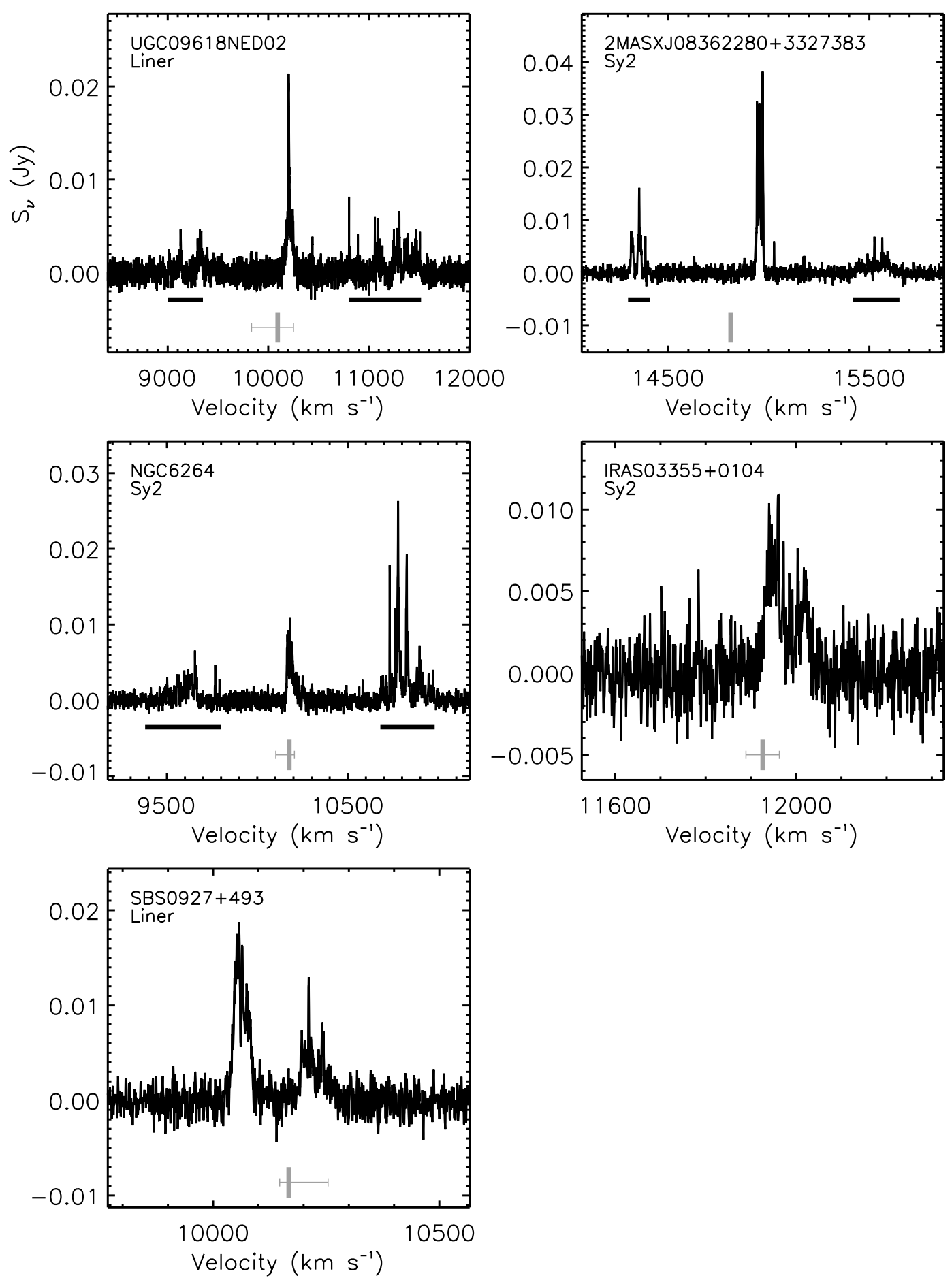

Fig. 1. - Spectra of UGC 09618 NED02, 2MASX J08362280+3327383, NGC 6264, IRAS 03355+0104, SBS 0927+493 obtained with the Green Bank Telescope. Vertical bars mark the optical heliocentric systemic velocities of the host galaxies listed in the NED, while the associated error bars show the range of listed systemic velocity estimates. Horizontal bars indicate approximate velocity ranges of high-velocity emission. To illustrate the difference in the velocity scale among spectra, the tick marks are placed every $100 \mathrm{~km} \mathrm{~s}^{-1}$ for each $\mathrm{x}$-axis. 

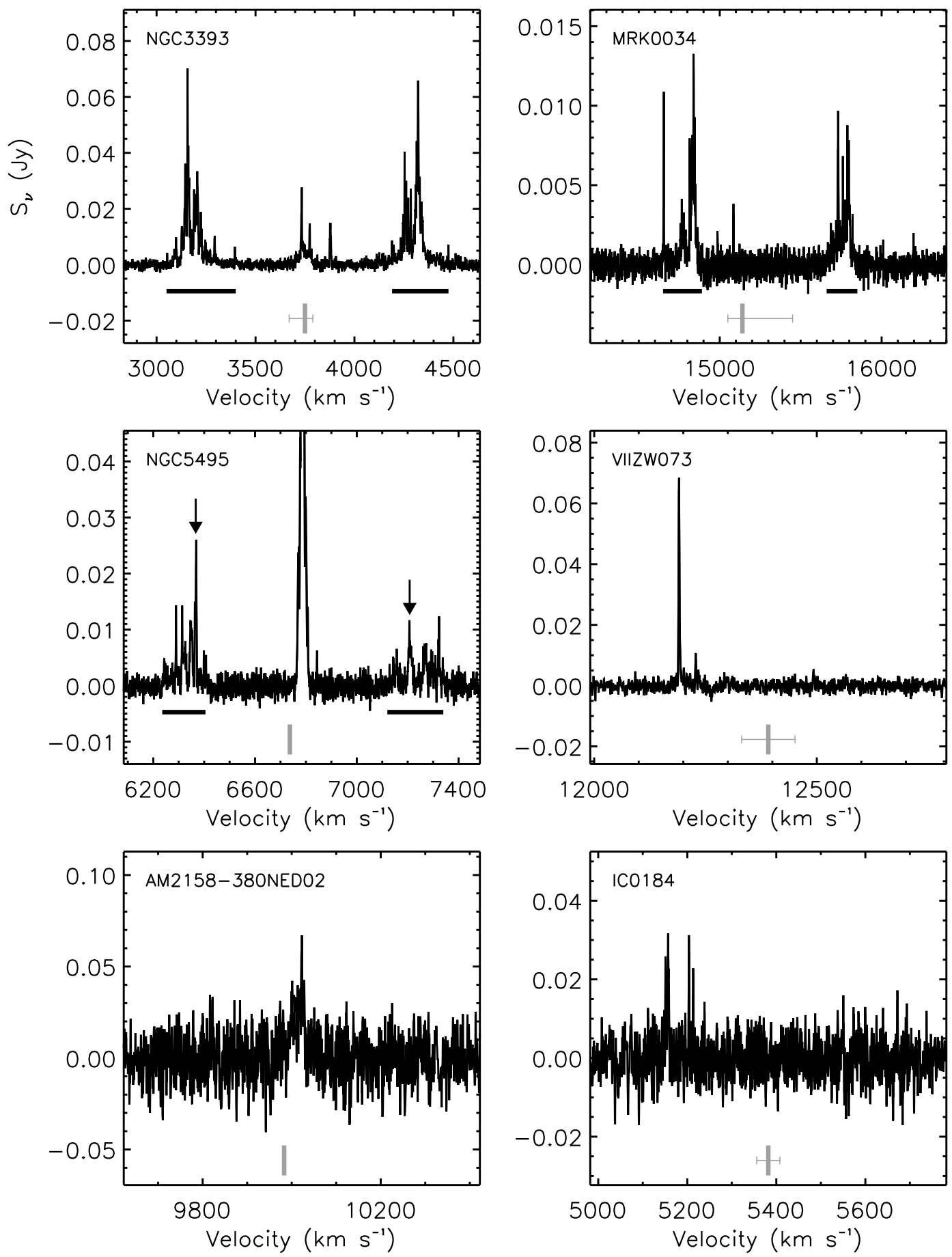

Fig. 2.- Spectra of previously known masers in NGC 3393, MRK 0034, NGC 5495, VIIZW 073, AM 2158-380 NED02, and IC 0184 obtained with the Green Bank Telescope. The arrows indicate NGC 5495 high-velocity features marginally detected with the DSN (Kondratko, P. T., et al. 2006). Vertical bars mark the optical heliocentric systemic velocities of the host galaxies listed in the NED, while the associated error bars show the range of listed systemic velocity estimates. Horizontal bars indicate approximate velocity ranges of high-velocity emission. To illustrate the difference in the velocity scale among spectra, the tick marks are placed every $100 \mathrm{~km} \mathrm{~s}^{-1}$ for each x-axis. 


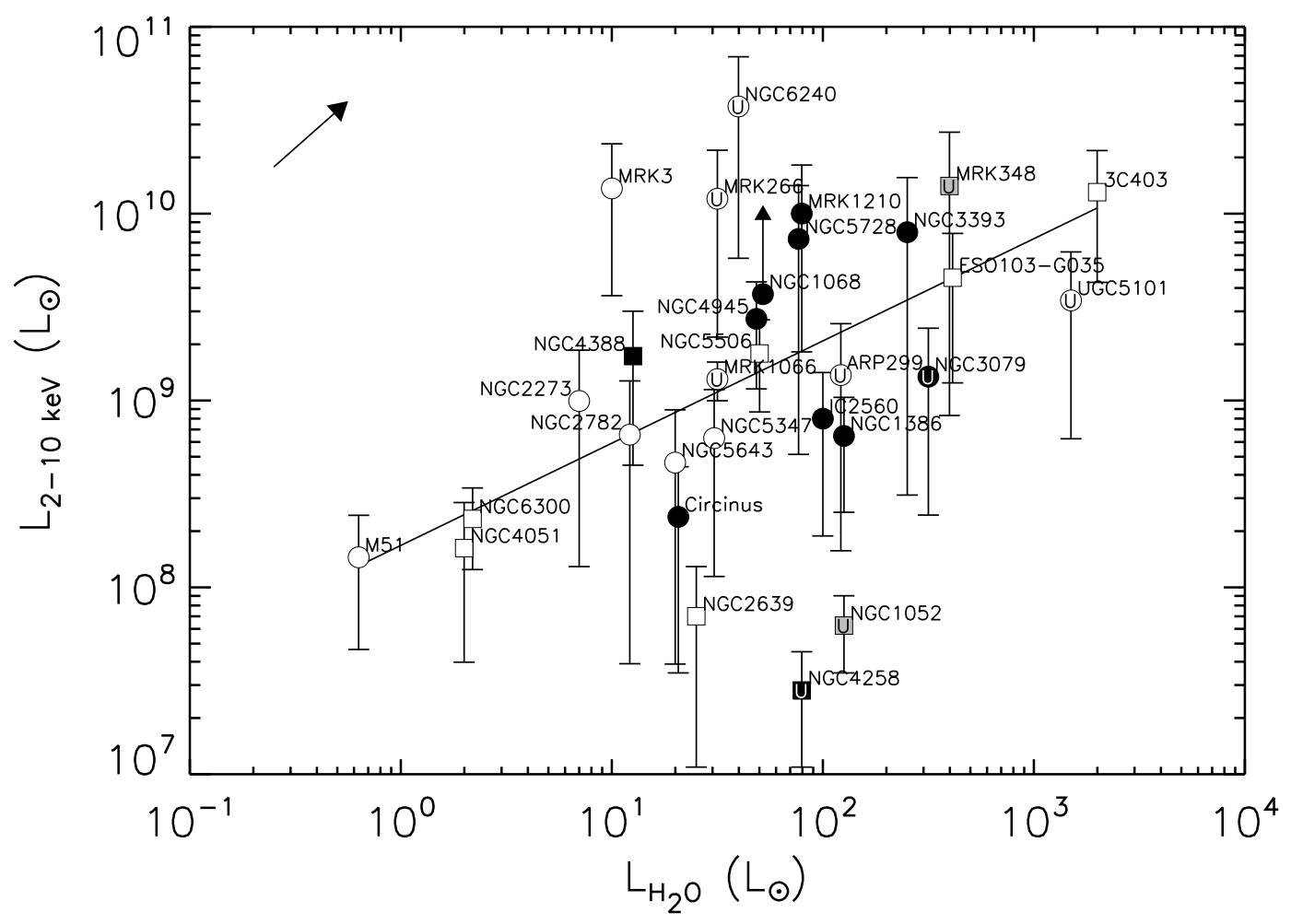

Fig. 3.- Unabsorbed X-ray luminosity $(2-10 \mathrm{keV})$ versus total isotropic water maser luminosity where the data points are from Table 4 . Symbol shading discriminates among maser types: (black) high-velocity systems (see definition in Section 1), (open) sources that are not known to be high-velocity systems, and (grey) maser sources known to be associated with radio jets rather than disks. Symbol shape differentiates between Compton-thick (round) and Compton-thin (square) objects. Galaxies with multiple nuclei, AGN with maser emission tied to jet activity, and LINER systems (see the text) are labelled by a letter U. Vertical error bars indicate the range of luminosity estimates given in the literature for independent data sets (Table 4), and the symbols are placed at the midpoints. The arrow in the top left corner illustrates the effect of a hypothetical factor of 1.5 increase in distance (i.e., points move up and to the right by this amount). For the full sample with no LINERs, systems with multiple nuclei, and "jet masers," we obtain a correlation coefficient of $0.5 \pm 0.1$ and a slope of $0.5 \pm 0.1$ (black line shows the results of a linear fit), where the asymmetric uncertainties in ordinate values have been taken into account (see Table 5). The lower limit on X-ray luminosity of NGC 1068 was not used in the fit. 